\title{
A espiritualidade no processo de formação dos profissionais de saúde
}

\author{
Spirituality in the training process of health professionals
}

La espiritualidad en el proceso de formación de los profesionales de salud

\author{
Annaterra Araújo Silva ${ }^{1 *}$, Agnes Claudine Fontes de La Longuiniere ${ }^{2}$, Ianderlei Andrade Souza ${ }^{3}$, \\ Amanda Sales Cafezeiro ${ }^{4}$, Ana Lúcia Gonçalves de Oliveira Cunha ${ }^{5}$, Sérgio Sonha Yarid ${ }^{6}$
}

Como citar esse artigo. Silva, AA; de La Longuiniere, ACF; Souza, IA; Cafezeiro, AS; Cunha, ALGO; Yarid, SS. A espiritualidade no processo de formação dos profissionais de saúde. Revista Pró-UniverSUS. 2020 Jul./Dez.; 11 (2): $158-163$.

\section{Resumo}

Introdução: A espiritualidade tem se mostrado um importante componente terapêutico no processo de restabelecimento da saúde dos indivíduos, mas, em razão da lacuna existente na formação acadêmica, ainda é pouco utilizada pelos profissionais de saúde em seus planos de intervenção. Destarte, o objetivo deste artigo é descrever como a espiritualidade foi abordada na formação acadêmica de profissionais de saúde. Materiais e Métodos: Estudo de caráter descritivo, transversal com abordagem quantitativa, mediante aplicação de questionário adaptado do estudo multicêntrico Spirituality and Brazilian Medical Education a 53 profissionais de saúde atuantes na Unidade de Terapia Intensiva Adulto de dois hospitais no interior da Bahia. Resultados: Aproximadamente $90 \%$ dos entrevistados relataram que na graduação não tiveram contato com o tema Espiritualidade/ Saúde, sendo que $81 \%$ gostariam de ter participado de alguma atividade relacionada ao tema e ainda, $70 \%$ afirmaram que este deveria fazer parte dos currículos dos profissionais de saúde. Discussão: A espiritualidade pode mudar a visão do profissional, evidenciando a real necessidade de uma formação reflexiva e pautada na universalidade do cuidado e na complexidade do ser humano que, para além de biológico, é psicológico, social e espiritual, sendo imprescindível sua inclusão na formação acadêmica do profissional de saúde. Considerações finais: As instituições de ensino não oferecem informações suficientes sobre o tema espiritualidade e saúde, de forma que os discentes que possuem interesse no assunto os adquirem e compartilham fora do espaço institucional.

Palavras-chave: Espiritualidade; Universidade; Graduação; Profissionais de Saúde.

\begin{abstract}
Introduction: Spirituality has been shown as an important therapeutic component in the process of recovering the individuals health, but, due to the gap in academic education, it is still little used by health professionals in their intervention plans. Thus, the aim of this article is to describe how spirituality was addressed in the academic training of health professionals. Materials and Methods: A descriptive, cross-sectional study with a quantitative approach, using a questionnaire adapted from the multicenter study Spirituality and Brazilian Medical Education to 53 health professionals working in the Adult Intensive Care Unit of two hospitals in the interior of Bahia. Results: Approximately $90 \%$ of the interviewees reported that at graduation they had no contact with the theme Spirituality / Health, and $81 \%$ would have liked to have participated in some activity related to the theme and yet, $70 \%$ stated that it should be part of the professionals' curricula of health. Discussion: Spirituality can change the professional's view, showing the real need for reflective training and guided by the universality of care and the complexity of the human being, which in addition to being biological, is psychological, social and spiritual, and its inclusion in the academic training of health professionals is essential. Final considerations: Educational institutions do not offer enough information on the topic of spirituality and health, so that students who have an interest in the subject acquire and share them outside the institutional space.

Keywords: Spirituality; University; Graduate; Health professionals.
\end{abstract}

Afiliação dos autores: ${ }^{~}$ Enfermeira. Mestre em Ciências da Saúde pelo Programa de Pós-Graduação em Enfermagem e Saúde da Universidade Estadual do Sudoeste da Bahia (UESB). Membro do Núcleo de Pesquisa em Bioética e Espiritualidade (UESB), Jequié/BA, Brasil. ORCID: https://orcid.org/0000-0001-7446-0446 ${ }^{2}$ Enfermeira. Doutoranda. Professora assistente da Universidade Estadual do Sudoeste da Bahia, Membro do Núcleo de Pesquisa em Bioética e Espiritualidade, Departamento de Saúde II da Universidade Estadual do Sudoeste da Bahia (UESB), Jequié/BA, Brasil. ORCID: https://orcid.org/0000-0003-2407-3338

${ }^{3}$ Cirurgião-dentista. Mestrado em Clínica Odontológica. Professor Assistente da Universidade Estadual do Sudoeste da Bahia (UESB). Membro do Núcleo de Pesquisa em Bioética e Espiritualidade (UESB), Jequié/BA, Brasil. ORCID: https://orcid.org/0000-0002-7270-8986

${ }^{4}$ Psicóloga. Membro do Núcleo de Pesquisa em Bioética e Espiritualidade da Universidade Estadual do Sudoeste da Bahia (UESB), Jequié/BA, Brasil. ORCID: https://orcid. org/0000-0003-1160-0872

${ }^{5}$ Advogada. Membro do Núcleo de Pesquisa em Bioética e Espiritualidade, Departamento de Saúde II da Universidade Estadual do Sudoeste da Bahia (UESB), Jequié/BA, Brasil. ORCID: https://orcid.org/0000-0001-8712-7183

${ }^{6}$ Cirurgião-dentista. Doutor em Odontologia Preventiva e Social pela UNESP. Professor Titular da Universidade Estadual do Sudoeste da Bahia (UESB). Coordenador do Núcleo de Pesquisa em Bioética e Espiritualidade (UESB), Jequié/BA, Brasil. E-mail: yarid@uesb.edu.br ORCID: https://orcid.org/0000-0003-0232-4212

* Email de correspondencia: annaterraraujo@live.com

Recebido em: 07/07/20. Aceito em: 18/10/20. 


\section{Resumen}

Introducción: la espiritualidad es un componente terapéutico importante en el proceso de restauración de la salud, pero, debido a la brecha en la educación académica, los profesionales de la salud todavía la utilizan poco en sus planes de intervención. Por lo tanto, el propósito de este artículo es describir cómo se abordó la espiritualidad en la formación académica de los profesionales de la salud. Materiales y métodos: un estudio descriptivo, transversal con un enfoque cuantitativo, utilizando un cuestionario adaptado del estudio multicéntrico Espiritualidad y Educación Médica Brasileña a 53 profesionales de la salud que trabajan en la Unidad de Cuidados Intensivos para Adultos de dos hospitales en el interior de Bahía. Resultados: Aproximadamente 90\% de los entrevistados informaron que en la graduación no tuvieron contacto con el tema Espiritualidad / Salud, y $81 \%$ le hubiera gustado haber participado en alguna actividad relacionada con el tema y, sin embargo, 70\% declaró que debería ser parte del plan de estudios de los profesionales. Discusión: La espiritualidad puede cambiar la visión del profesional, mostrando la necesidad real de capacitación reflexiva y guiada por la universalidad del cuidado y la complejidad del ser humano, que además de ser biológico, es psicológico, social y espiritual, y su inclusión en el Formación académica de profesionales de la salud. Consideraciones finales: las instituciones educativas no ofrecen suficiente información sobre el tema de la espiritualidad y la salud, por lo que los estudiantes que tienen un interés en el tema adquieren y comparten fuera del espacio institucional.

Palabras clave: Espiritualidad; Universidad; Graduado universitario; Profesionales de la salud.

\section{Introdução}

A espiritualidade tem sido considerada um importante componente terapêutico no processo de restabelecimento da saúde dos indivíduos, bem como uma dimensão fundamental para o cuidado ao paciente em sua integralidade. ${ }^{1}$ Apesar disto, permanece pouco abordada por profissionais de saúde durante a assistência, pois estes nem sempre receberam em suas formações preparo adequado para inclui-la em seus planos de intervenção.

A Organização Mundial da Saúde (OMS) inclui a dimensão espiritual na definição multidimensional de saúde, que passou a reunir aspectos biopsicossociais e espirituais. Desta forma, convicções e vivências espirituais passaram a ser elementos significativos ao cuidado integral, sendo reconhecida tal relevância também por pesquisadores, profissionais e usuários dos serviços de saúde. Nessa conjuntura, a espiritualidade e a religiosidade tornam-se mecanismos importantes da assistência à saúde ${ }^{2}$.

Ao levar-se em consideração os cuidados paliativos, estudos indicam que fatores relacionados à espiritualidade ajudam na promoção do bem-estar frente às doenças, prevenção e na recuperação dos indivíduos que sofrem desequilíbrios orgânicos ou psíquicos ${ }^{3}$. Mas esta abordagem pelas escolas de formação brasileiras ainda é pequena, conforme demonstram estudos onde apenas uma pequena parcela de profissionais médicos discute a espiritualidade com seus pacientes, pois os mesmos referem não ter sido preparados para aprofundar o cuidado levando em consideração tal aspecto ${ }^{4}$.

Existe grande potencial terapêutico quando os profissionais formados atentam à condição do ser humano de uma forma integral. Esta conduta profissional atinge diretamente a relação entre o médico e o paciente podendo influenciar em todas as fases do processo de cuidar. Desta forma, numa visão integral sobre o indivíduo devem estar embutidos seus propósitos de vida, valores e concepção de mundo ${ }^{5}$, o que pode incluir a espiritualidade, tendo em vista que esta é inerente ao ser e refere-se ao seu íntimo, suas acepções e compreensões de mundo, na busca de ligações com o próximo, com o universo e com um ser superior, tendo associação a um significado religioso ou não ${ }^{6}$.

A dimensão espiritual ganha notória importância em cenários críticos, nos quais os indivíduos são confrontados com questões fundamentais do existir humano, como a morte. Isto pode ser constatado em Unidades de Terapia Intensiva (UTI), por exemplo: locais de alta complexidade, que atendem pacientes graves no intuito de reestabelecer a saúde. A gravidade dos quadros de saúde dos pacientes nela internados convoca maior atenção não só aos mesmos, mas à toda a equipe multiprofissional que passa por situações lime durante a rotina diária na UTI, o que pode interferir negativamente em seu modo de viver e, principalmente, de cuidar?

É corriqueiro que pacientes e familiares diante dos sentimentos vindos com o adoecer como sofrimento, dor, medo, desesperança, sensação de finitude da vida recorram à espiritualidade como busca de conforto e apoio. A espiritualidade se torna de grande importância como parte da assistência integral à saúde que os profissionais de uma UTI devem proporcionar aos pacientes e familiares que estão passando por situações tão delicadas ${ }^{8}$.

A potencialização de um cuidado humanizado e integral dos profissionais de enfermagem é observada no movimento entre cuidar e cuidar-se em Unidade de Terapia Intensiva, contudo, o modelo assistencialista adotado que costuma ser rigoroso nas padronizações, não permite a exposição desses sentimentos durante a assistência, levando à equipe ao sofrimento psicológico, principalmente por estarem expostos a atmosferas de dor, sofrimento e perdas 9 .

Portanto, é necessário que nas instituições de ensino superior seja discutida no processo formativo a dimensão espiritual como elemento da assistência à saúde relevante para o processo saúde-doença do paciente, além da possibilidade de tornar o cuidado humanizado, integral e ético ${ }^{10}$. Assim, o objetivo deste estudo foi descrever como ocorreu a abordagem da 
espiritualidade na formação acadêmica de profissionais de saúde, tendo sido selecionado como campo de pesquisa os profissionais de saúde de Unidade de Terapia Intensiva, por estarem mais expostos à cenários críticos e de grande vulnerabilidade humana.

\section{Método}

Estudo de caráter descritivo, transversal com abordagem quantitativa. Foi realizado em uma UTI Adulto de Hospital Geral Público e numa UTI Adulto de Hospital privado.

Do total de 86 profissionais, 53 aceitaram participar da pesquisa onde todos prestavam assistência direta ao paciente grave nas Unidades de Terapia Intensiva. Participaram desta pesquisa enfermeiros, médicos, técnicos de enfermagem, fisioterapeutas e psicólogos. Foram utilizados como critérios de inclusão: ser profissional de saúde, prestar assistência em regime de plantão ao paciente grave nas Unidades de Terapia Intensiva do estudo e estar em atividade no serviço, neste período. Foram excluídos desta pesquisa àqueles que trabalhavam na UTI, mas que não eram profissionais da saúde (profissional de higienização, secretária), profissionais que estavam de férias ou licença médica no período da coleta e àqueles profissionais que apenas assistiam eventualmente o paciente (inter -consulta de especialidades médicas).

Como instrumento de coleta de dados foi utilizado um questionário adaptado do estudo Multicêntrico Spirituality and Brazilian Medical Education, coordenado pela Universidade Federal de São Paulo (UNIFESP), Universidade Federal de Juiz de Fora e pela Associação Médico Espírita do Brasil ${ }^{11}$.

O questionário utilizado foi composto pelas seguintes questões: dados sociodemográficos: gênero, idade, tempo de serviço, raça/etnia e renda familiar; prática clínica: o paciente e a espiritualidade; formação acadêmica e o tema espiritualidade; e dimensão de religiosidade ${ }^{12}$. Para este estudo foram utilizados apenas os dados referentes à categoria de "formação acadêmica e o tema espiritualidade".

Os profissionais foram convidados a participar do estudo durante o período do plantão e após explicação sobre os objetivos da pesquisa, seus riscos e relevância, aqueles que aceitaram participar, assinaram o Termo de Consentimento Livre e Esclarecido. Os questionários foram aplicados no período de 01 de março a 30 de abril de 2015 durante o plantão nas Unidades de Terapia Intensivas.

Após a coleta, os dados foram tabulados no programa Microsoft Excel e, posteriormente, transferidos para o programa estatístico SPSS versão 21.0. Realizada análise descritiva dos dados mediante a distribuição de frequência absoluta, e relativa para as variáveis categóricas e medidas de tendência central (média, mediana e desvio padrão) para as variáveis numéricas.

A pesquisa foi realizada seguindo a Resolução 466/12 do Conselho Nacional de Saúde ${ }^{13}$, que aborda os aspectos éticos da pesquisa envolvendo seres humanos, pautada nos princípios básicos da bioética. A pesquisa foi aprovada no Comitê de Ética em Pesquisa da Universidade Estadual do Sudoeste da Bahia com CAAE 32197814.90000.0055sob o número de parecer 805.380.

\section{Resultados}

Participaram desta pesquisa 53 profissionais de saúde que atuavam em UTI. Destes, $77 \%$ eram do sexo feminino, a maioria tinha cor auto- referida não branca, renda mensal familiar até 7 salários mínimos, idade entre os 26 e 63 anos, com idade média de 37 anos e religião evangélica. O tempo de serviço variou entre 2 a 29 anos, com tempo médio de 11 anos de assistência à saúde de 4 anos de experiência em UTI, conforme tabela 1.

Sobre a abordagem de crenças religiosas ou espirituais nas atividades curriculares durante a graduação, $57 \%$ dos entrevistados relataram que os docentes abordaram este tema com pouca frequência e $37 \%$ referem que nunca estudaram o tema e $6 \%$ não responderam.

A maioria dos participantes (88\%) também referiu que a instituição de formação não forneceu informações suficientes para que estes, enquanto profissionais de saúde, pudessem abordar sobre as crenças religiosas ou espirituais dos pacientes.

Quando questionado se o profissional participou de alguma atividade durante a formação sobre "Saúde e Espiritualidade", aproximadamente $90 \%$ responderam "não". Dentre estes, $81 \%$ informaram que gostariam de ter participado, sendo que, para $70 \%$ dos profissionais entrevistados, temas relacionados à "Saúde e Espiritualidade" deveriam fazer parte dos currículos dos profissionais de saúde.

Ao avaliar como o conhecimento sobre espiritualidade relacionado à saúde é adquirido, 40\% responderam que procuram ensinamentos dentro da própria religião, 36\% referem leituras de livros, artigos, palestras ou que recorria aos docentes, assim como $24 \%$ não costumam buscar conhecimentos sobre o tema.

\section{Discussão}

A complexidade do cuidado, principalmente daqueles pacientes em estado grave, nas UTIs tem exigido cada vez mais conhecimento técnico científico para a oferta da assistência, bem como a se observa que a formação profissional ainda está baseada no modelo 
Tabela 1. Características sócio demográficas e religião praticada por profissionais que atuam em Unidade de Terapia Intensiva. Jequié, 2015.

\begin{tabular}{|c|c|c|c|c|}
\hline VARIÁVEL & $\mathbf{N}(\%)$ & MÉDIA & MEDIANA & DP \\
\hline Idade (anos) & $53(100)$ & 37,11 & 35,00 & 8,07 \\
\hline Tempo de serviço (anos) & $53(100)$ & 11,12 & 8,50 & 7,07 \\
\hline Tempo de UTI (anos) & $53(100)$ & 4,38 & 4,00 & 2,97 \\
\hline \multicolumn{5}{|l|}{ Gênero } \\
\hline Masculino & $12(22,6)$ & - & - & - \\
\hline Feminino & $41(77,4)$ & - & - & - \\
\hline \multicolumn{5}{|l|}{ Cor de pele auto referida } \\
\hline Branco & $17(32,1)$ & - & - & - \\
\hline Não Branco & $36(67,9)$ & - & - & - \\
\hline \multicolumn{5}{|l|}{ Renda } \\
\hline Até 7 salários mínimos & $35(66)$ & - & - & - \\
\hline Acima de 7 salários mínimos & $18(34)$ & - & - & - \\
\hline \multicolumn{5}{|l|}{ Religião } \\
\hline Apenas acredita em Deus & $4(7,5)$ & - & - & - \\
\hline Evangélico/Protestante & $19(35,9)$ & - & - & - \\
\hline Espírita & $7(13,2)$ & - & - & - \\
\hline Católico & $18(34,0)$ & - & - & - \\
\hline Espiritualista & $3(5,7)$ & - & - & - \\
\hline Outros & $2(3,7)$ & - & - & - \\
\hline
\end{tabular}

Fonte: Fonte: Dados da Pesquisa, 2015.

reducionista organicista da medicina vigente. Esse modeloestá fortemente focadonacura do corpobiológico, privilegiando a doença e não a pessoa adoecida, o que contribui para a formação de profissionais de saúde que não valorizam a assistência voltada ao binômio saúdedoença, no qual os aspectos psíquicos e físicos são indissociáveis para o restabelecimento do equilíbrio ${ }^{14}$.

Muitos profissionais não visualizam os limites da utilização da fé como recurso para estabelecimento de um vínculo terapêutico diante dos pacientes e familiares. Isto se torna importante pois a qualidade das relações é primordial para o cuidado efetivo, portanto o sistema de crença tem relação direta na formação do vínculo de cuidado e se tiver interferência negativa nos relacionamentos interpessoais que inviabilize ou dificulte o contato, pode levar a um grande problema assistencial ${ }^{15}$.

Diante da comprovação de que a aprendizagem da dimensão espiritual envolve conceitos éticos e morais que são essenciais para lidar com a integralidade do indivíduo ${ }^{16}$, este estudo demonstra que $57 \%$ dos entrevistados informaram que o tema espiritualidade foi abordado com pouca frequência pelos docentes, levando ao despreparo dos profissionais ao lidar com a dimensão espiritual dos pacientes e $37 \%$ referem que nunca estudaram o tema,

Embora a maioria das universidades brasileiras ainda não disponibilize a espiritualidade e saúde como disciplina, alguns estudos trazem que ao longo do curso os estudantes não receberam subsidio necessário para enfrentarasinquietações espirituais vividas pelopaciente. Enfatiza-se que mesmo que o atendimento espiritual seja um recurso terapêutico de grande importância na assistência aos pacientes, sua execução é negligenciada pelos profissional de saúde ${ }^{17}$, o que corrobora com o estudo, onde a maioria dos participantes $(88 \%)$ também referiu que a instituição não disponibiliza informações suficientes para que estes, enquanto profissionais de saúde, pudessem abordar sobre as crenças religiosas ou espirituais dos pacientes.

É notório a não existência de disciplinas específicas sobre Espiritualidade/Saúde nas instituições de ensino apesar das mesmas reconhecerem a falta de preparo e à dificuldade de atender aos problemas espirituais do paciente, mostrando a falta de conhecimento e informações sobre o tema ${ }^{6,18}$ sobre o tema. No entanto, não é oferecido um ensino de modo substancial e detalhado da espiritualidade e sua relação com a saúde mental e física ${ }^{19}$. Confirmando, pode-se observar que, aproximadamente $90 \%$ dos entrevistados não tiveram contato com o assunto, sendo que $81 \%$ dentre eles gostariam de ter participado de alguma atividade relacionada ao tema Espiritualidade/Saúde e ainda, $70 \%$ afirmaram que este deveria fazer parte dos 
currículos dos profissionais de saúde.

Alguns profissionais expressam dificuldade em abordar a espiritualidade dos seus pacientes, pois consideram somente o aspecto religioso dos enfermos, sendo que conceitualmente a $\mathrm{R} / \mathrm{E}$ são diferentes, encontrando desta forma obstáculos no modo de abordar as crenças e oferecer apoio espiritual aos pacientes, com o objetivo de proporcionar conforto e bem-estar para aquele que sofre, sendo que também há referência sobre a falta de tempo para se dedicar aos momentos particulares dos pacientes, além da sobrecarga de trabalho.

Nessa pesquisa, ficou evidenciado que $40 \%$ dos entrevistados procuram ensinamentos dentro da própria religião. Em um estudo feito sobre a influência da R/E no contexto psicoterapêutico de profissionais mostra que, em muitas Universidades, a dimensão religiosa e espiritual estava relacionada a diversas doenças e distúrbios mentais, pois havia a crença que a religião era tóxica à saúde mental ${ }^{20}$.

Ser espiritualizado é de suma importância para o profissional de saúde ao lidar com temas delicados, pois a espiritualidade seria uma forma de buscar o sentido da vida, da doença, da morte ou do sofrimento, além de ser um processo muito importante para aqueles momentos difíceis durante a internação e o adoecimento, podendo favorecer o amadurecimento pessoal e trazer um melhor enfrentamento da situação ${ }^{21}$.

Alguns estudiosos ressaltam que a espiritualidade pode mudar a visão do profissional, evidenciando a real necessidade de uma formação profissional reflexiva e pautada na universalidade do cuidado e na complexidade do ser humano ${ }^{22}$. Dessa forma, é importante ressaltar que, ao prestar o cuidado, o profissional deve compreender que a espiritualidade é inerente, específica e particular de cada ser, mas é preciso que ele se reconheça como um ser biológico, psicológico, social e espiritual, buscando descobrir e progredir sua transcendência como ser humano ${ }^{23}$

\section{Considerações Finais}

Apesar de a espiritualidade ser apontada por estudos como instrumento que colabora com o cuidado na promoção e recuperação da saúde, notou-se que a abordagem da espiritualidade na formação acadêmica de profissionais de saúde ocorre de maneira insuficiente. Isto aponta para a necessidade de uma reflexão acerca do processo de formação que deve considerar o ser humano em suas diversas dimensões durante o processo de cuidar.

Os profissionais de saúde que atuam com pacientes críticos nas UTIs deste estudo referiram que a instituição de formação não forneceu informações suficientes para que eles abordassem a dimensão espiritual dos pacientes. Apesar de quase $90 \%$ não terem participado de atividades sobre a temática durante a graduação, a maioria destes reconheceram que este tema deveria fazer parte do currículo dos profissionais de saúde.

Assim, estudos como este poderão sensibilizar a comunidade acadêmica quanto à necessidade de incluir a espiritualidade na formação dos profissionais de saúde, contribuindo para um modelo contemporâneo do cuidado, compreendendo o ser humano que está doente em suas variadas dimensões e vulnerabilidades no enfrentamento da doença.

\section{Referências}

1. Evangelista CB, Lopes MEL, Costa SFG, Batista PSS, Batista JBV, Oliveira AMM. Palliative care and spirituality: an integrative literature review. Rev Bras Enferm [Internet]. 2016;69(3):554-63. DOI: http://dx.doi. org/10.1590/0034-7167.2016690324i

2. Abdala GA, Meira MDD, Oliveira SLS, Santos DC. Religión, espiritualidad y enfermería. REFACS (online) [periódico na Internet]. 2017 [acesso em 2017 Fev 13];5(1): [aproximadamente 10 p.]. Disponível em: $\quad$ http://seer.uftm.edu.br/revistaeletronica/index.php/refacs/article/ viewFile/2001/2042. DOI: http://dx.doi.org/10.18554/refacs.v5i0.2001.

3. CullifordL. Spiritualityandclinicalcare.BMJ.2002;325(7378):1434-5. DOI: http://dx.doi.org/10.1136/bmj.325.7378.1434.

4. Lucchetti G, Lucchetti ALG, Espinha DCM, Oliveira LR, Leite JR, Koenig HG. Spirituality and health in the curricula of medical schools in Brazil. BMC Medical Education [periódico na Internet]. 2012 [acesso em 2017 Maio 26];12(78): [aproximadamente 8 p.]. Disponível em: https:// bmcmededuc.biomedcentral.com/articles/10.1186/1472-6920-12-78. DOI: https://doi.org/10.1186/1472-6920-12-78

5. Puchalski CM, Blatt B, Kogan M, Butler A. Spirituality and health: the development of a field. Acad Med. 2014;89(1):1-7. DOI: http://dx.doi. org/10.1097/ACM.0000000000000083.

6. Ferreira AGC, Oliveira JAC, Jordán APW. Spirituality and health education: a proposal for

7. transversality in the student's perspective. Interdisciplinary Journal of Health Education, 2016; 1(1):3-12. DOI: 10.4322/ijhe2016005.

8. Santos RB, Duran ECM, Carmona EV. et al. Nursing diagnoses in patients with hospital infection. Revista enfermagem UFPE online [periódica internet] Recife. 2015 Set [acesso em 2018 Ago 12];9(8):[aproximadamente 7 p.]. Disponível em: https://periodicos.ufpe.br/revistas/revistaenfermagem/ article/download/10741/11849. DOI: 10.5205/reuol.6812-75590-1ED.0908sup201504.

9. Pilger C, Macedo JQ, Zanelatto R, Soares LG, Kusumota L. Percepção da equipe de enfermagem de uma unidade de terapia intensiva com relação à espiritualidade e religiosidade. Cienc Cuid Saude. 2014 Jul-Set; 13(3):479486. DOI: 10.4025/cienccuidsaude.v13i3.19788.

10. Penha RM, Silva MJP. Significado de espiritualidade para a enfermagem em cuidados intensivos. Texto contexto - enferm. [Periódico na Internet]. 2012 Jun [acesso em 2018 Ago 27];21(2):260-268. Disponível em: http://www.scielo.br/scielo.php?script=sci arttext\&pid=S01047072012000200002\&lng=en. $\quad$ DOI: http://dx.doi.org/10.1590/S010407072012000200002 .

11. Ermel RG, Vieira M, Tavares TF, Furuta PM, Zutin TL, Caramelo AC The spiritual well-being of the faculty of medicine and nursing. Rev enferm UFPE online [periódico na Internet]. Recife. 2015 Jan [acesso em 2017 Maio 25];9(1):[aproximadamente 11 p.]. Disponível em: http://www.revista.ufpe. br/revistaenfermagem/index.php/revista/article/viewArticle/5303. DOI: 10.5205/reuol.6817-60679-1-ED.0901201522.

12. Lucchetti G, Oliveira LR, Koenig HG, Leite JR, Lucchetti ALG, and for the SBRAME (Collaborators). Medical Students, Spirituality and Religiosity-results from the Multicenter Study SBRAME.BMC Medical Education [periódico na Internet]. 2013 [acesso em 2016 Set 30];13(162): 
[aproximadamente $8 \mathrm{p}$.]. Disponível em: http://www.biomedcentral. com/1472-6920/13/162.

13. Lucchetti G, Granero Lucchetti AL, Peres MF, Leão FC, MoreiraAlmeida A, Koenig HG. Validation of the duke religion index: DUREL (portuguese version). J Relig health [periódico na Internet]. 2012 Jun [acesso em 2016 Set 30];51(2): [aproximadamente 8 p.]. Disponível em: https://www. ncbi.nlm.nih.gov/pubmed/21107911. DOI: 10.1007/s10943-010-9429-5.

14. Brasil. Conselho Nacional de Saúde. Resolução $\mathrm{n}^{\circ} 466$, de 12 de dezembro de 2012. Aprova normas regulamentadoras de pesquisas envolvendo seres humanos. Brasília: Diário Oficial da União. 2013. Disponível em: http://conselho.saude.gov.br/resolucoes/2012/reso466.pdf.

15. Silva JB, Aquino TA, Silva AF. As relações entre espiritualidade e cuidado segundo as concepções de estudantes de enfermagem. Rev enferm UFPE online [periódico na Internet]. Recife. 2016 Mar [acesso em 2017 Maio 24];10(3): [aproximadamente 9 p.]. Disponível em: http://www.revista. ufpe.br/revistaenfermagem/index.php/revista/article/view/7152/pdf 9848 . DOI: 10.5205/reuol.8702-76273-4-SM.1003201612.

16. Koenig H. Religious attitudes and practices of hospitalized medically ill older adults. Int J Geriatr Psychiatry. 1998 Abr;13(4):213-24. DOI: 10.1002/(SICI)1099-1166(199804)13:43.0.CO;2-5.

17. Marques LF. Religiosidade/espiritualidade na educação e na saúde: ensino e extensão. Rev. Pistis Prax. Teol. Pastor. Curitiba. 2017 JanAbr;9(1):189-203. DOI: http://dx.doi.org/10.7213/2175-1838.09.001.DS09.

18. Balboni T, Balboni M, Paulk Me, Phelps A, Wright A, Peteet J, et al. Support of cancer patients' spiritual needs and associations with medical care costs at the end of life. Cancer [periódico na Internet]. 2011 [acesso em 2018 Ago 27];117(23):[aproximadamente 9 p.]. Disponível em: http://www.ncbi. nlm.nih.gov/pubmed/21563177. DOI: 10.1002/cncr.26221.

19. Tomasso CS, Beltrame IL, Lucchetti G. Knowledge and attitudes of nursing professors and students concerning the interface between spirituality, religiosity and health. Revista Latino-Am Enfermagem [periódico na Internet]. 2011 [acesso em 2018 Ago 28];19(5):[aproximadamente 9 p.]. Disponível em: http://www.scielo.br/pdf/rlae/v19n5/19.pdf. DOI: http:// dx.doi.org/10.1590/S0104-11692011000500019.

20. Freitas MF, Piasson DL. Religião, religiosidade e espiritualidade: repercussão na mídia e formação profissional em psicologia. Esferas. 2016 Jan-Jun;5(8):103-12. DOI: http://dx.doi.org/10.19174/esf.v1i8.7909.

21. Henning-Geronasso MC, Moré CLOO. Influência da Religiosidade/ Espiritualidade no Contexto Psicoterapêutico. Psicologia: Ciência e Profissão, 2015;35(3):711-725. DOI: http://dx.doi.org/10.1590/1982-3703000942014.

22. Manenti LP, Soratto MT. A importância da espiritualidade no cuidado com o paciente internado na UTI cardiovascular. Saúde em Revista. Piracicaba, 2012 Jan-Abr;12(30):43-51. DOI: http://dx.doi.org/10.15600/2238-1244/ sr.v12n30p43-51.

23. Reginato V, Benedetto MAG, Gallian DMC. Espiritualidade e saúde: uma experiência na graduação em medicina e enfermagem. Trab. Educ. Saúde. Rio de Janeiro. 2016 Jan-Abr;14(1):237-255. DOI: http://dx.doi. org/10.1590/1981-7746-sip00100.

24. Barros Júnior J, Barbosa RS, Luna Neto RT, Tavares NBF, Freitas KM, Cruz CA, et al. Saúde e Espiritualidade: As Narrativas Profissionais na Estratégia Saúde da Família. Revista de psicologia [periódico na Internet]. 2015 Abril [acesso em 2017 Fev 12];9(26): [aproximadamente 11 p.]. Disponível em: https://idonline.emnuvens.com.br/id. DOI: https://doi. org/10.14295/idonline.v9i26.329. 